\title{
EL CONCEPTO DE INTEGRIDAD EN DWORKIN*
}

\section{Introducción}

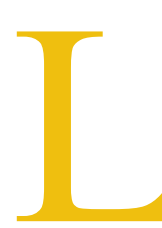

a obra de Dworkin ha originado una polémica importante en la historia de la filosofía político-jurídica ${ }^{1}$. No es frecuente que los trabajos de un filósofo del derecho despierten el interés y la crítica de constitucionalistas, sociólogos, filósofos, economistas, politólogos o estudiosos de la ética. Sin duda, tiene muchos más detractores que defensores. Uno de sus méritos incuestionables ha sido provocar la discusión más allá de sus límites gremiales y académicos para extenderse a ámbitos pluridisciplinarios y llegar a públicos que en principio no están interesados en los problemas específicos de los juristas tal y como se han tratado en los círculos de especialistas.

No me propongo analizar todos y cada uno de los grandes temas que sugiere provocativamente Dworkin. Quisiera señalar que, del análisis de la crítica al modo de construir la ciencia jurídica tradicional, surge un nuevo modelo de construcción del saber jurídico en el cual el análisis del desacuerdo entre los juristas, el de los materiales mediante los cuales construye sus

* He recibido las ayudas PB 90-0327 y BE/ 92/ 39 del programa sectorial de Promoción General del Conocimiento de la DGTYC. Debo agradecer los comentarios y críticas de los profesores Raz, Garzón, Chang, de Lucas, Moreso y el equipo de profesores de la Universidad Pompeu Fabra.

${ }^{1}$ Véase M. Cohen, Ronald Dworkin and Contemporary Jurisprudence. London, Duckworth, 1984. Véase también R. Dworkin, Taking Rights Seriously. Harvard University Press, 6 ed., 1977 especialmente el apéndice A Reply to my Critics pág. 290 y ss. Acaban de aparecer dos libros importantes sobre Dworkin, S. Guest., R. Dworkin. Edimburg University Press 1992, y A. Hunted ed., Reading Dworkin Critically. Berg Publishers 1992. En la literatura castellana véase los trabajos de G. Carrió, Principios Jurídicos y Positivismo Jurídico. Buenos Aires, Abeledo Perrot, 1970 y «Le opinioni del Prof. Dworkin sul positivismo giuridico» en Materiali per una Storia della Cultura Giuridica, 1980. 
argumentos y el modelo de una sociedad democrática son a mi juicio los más relevantes. En este trabajo voy a seguir el siguiente esquema: En primer lugar, explicaré por qué ha tenido tanto éxito su obra. En segundo lugar, me preguntaré por el paradigma de la ciencia jurídica y sus límites. En tercer lugar, analizaré la estrategia elegida por Dworkin basada en los desacuerdos teóricos. Ello sugerirá la idea de que el derecho no es un producto acabado, sino un enfoque. En cuarto lugar -siguiendo el hilo argumentativo dworkiniano- nos preguntaremos hasta qué punto el pasado determina el presente y veremos que las doctrinas ofrecen respuestas contradictorias. Por último, nos plantearemos el modelo valorativo de una sociedad democrática y el papel de los principios y el equilibrio necesario entre ellos.

\section{La importancia de Dworkin}

Quienquiera que lea las principales revistas de Filosofía del Derecho y de la Política observará el éxito del «fenómeno Dworkin». Algunos pueden pensar que se debe al excelente marketing del autor, profesor en Oxford y New York, conversador y discutidor incansable, con una formación jurídica sólida y con una brillantez fuera de lo común en la construcción de contraejemplos que destruyen los argumentos de sus críticos. Además de eso, nuestro autor tiene una gran capacidad para introducir continuamente distinciones conceptuales que producen cuando menos la perplejidad o la desorientación del crítico. A todo ello se añade su continuo viajar para discutir y reformular sus posiciones. Escritor prolífico y comentador de la política del día desde las páginas del New York Book Review. Todo eso es cierto y dota de fundamento a la idea de que en el fondo sus planteamientos tienen un excelente sistema de difusión, pero no son tan originales, pues muchas de sus ideas ya se habían planteado por otros autores. Creo que esta idea no es exacta. Argumentaré que nos encontramos ante una obra sugerente que aprovecha viejas ideas pero en un contexto totalmente novedoso.

Pero no sólo eso. Su éxito se debe -desde mi punto de vista- sobre todo a la utilización de un método de análisis del derecho que permite no sólo describirlo sino también proponer un enfoque consistente de los problemas y apuntar soluciones sustentadas en un conjunto de principios coherentes. Para lograr este objetivo ha tenido que utilizar armas críticas y defender posiciones muy heterodoxas en una época en que tanto la filosofía jurídica analítica de Hart como la filosofía política utilitarista constituían las doc- 
trinas dominantes. A estas doctrinas ha dedicado sus dardos críticos más espectaculares (Dworkin, 1986, págs. 288-295; 1977, págs. 94 y ss. y 1985, págs. 81 y ss.).

\section{La crítica al modelo positivista de ciencia jurídica}

\section{La concepción del derecho como un producto acabado}

Dworkin es radical porque cuestiona el paradigma austiniano sobre el cual se ha construido la ciencia jurídica anglosajona del siglo XIX y XX (MacCormick, 1984, págs. 128 y ss.). Austin sostuvo que una ciencia completa del derecho debía tratar dos problemas fundamentales. Por una parte la Ciencia de la Legislación se ocupaba de cómo debe ser el derecho. La Ciencia de la Legislación es una disciplina prescriptiva y sus cultivadores son los políticos y filósofos. Junto a la Ciencia de la Legislación existe otra disciplina -la Jurisprudencia-, cuyo objeto es la descripción del derecho positivo. La Jurisprudencia responde a la cuestión de cómo es el derecho. Ésta es la disciplina típicamente jurídica cultivada por los juristas. El paradigma positivista no sólo exigió la distinción entre las disciplinas, sino que sostuvo que no existían puentes entre la Jurisprudencia y la Ciencia de la Legislación ${ }^{1 b i s}$. Para describir el derecho no es necesario saber cómo debe ser. La ciencia jurídica debe limitarse a la descripción del derecho positivo prescindiendo de los juicios de valor. La ciencia jurídica debe ser neutral y por tanto independiente de los intereses políticos. El único puente de comunicación va de la Jurisprudencia a la Ciencia de la Legislación, pues para diseñar cómo debe ser el derecho es preciso reunir información sobre el derecho positivo. Sin embargo, los filósofos y políticos no han tenido muy en cuenta esta limitación, ya que la mayoría de los proyectos de una sociedad mejor se dibujan en hojas en blanco sin tener en cuenta cómo es la sociedad y el derecho actual (Dworkin, 1986, pág. 164). En este sentido la mayoría de las filosofías políticas son utópicas. Dworkin pretende construir su filosofía jurídica -como la columna vertebral del razonamiento jurídico- desde una perspectiva no utópica, pues afirma: «la filosofía jurídica no es una disciplina de segundo orden que tenga como objeto el razonamiento ordinario, sino que

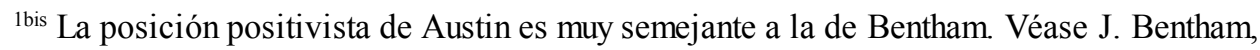
An Introduction to the Principles of Morals and Legislation. Ed. Burns y Hart. London University, Athlone 1970, págs. 293 y ss., en donde establece la distinción entre censorial and expository jurisprudence. 
ella misma es el nervio de la reflexión sobre el derecho» (Dworkin, 1980, pág. 8).

El punto de vista de Austin y de sus seguidores supone que el derecho es un producto acabado y que el científico del derecho tiene un objetivo fundamental: describir y explicar ese derecho. El científico es un descriptor de ese producto. Las teorías jurídicas de Kelsen y Hart están en esa línea y constituyen las versiones más sofisticadas del paradigma austiniano.

\section{La concepción del derecho como una actividad de justificación}

La osadía de Dworkin consiste en poner en cuestión ese paradigma. Pretende restaurar las relaciones entre la Ciencia de la Legislación y la Jurisprudencia poniendo de manifiesto que la tarea de la ciencia jurídica no es describir el derecho desde fuera, sino ofrecer solución a los problemas que se plantean. Pretende, por tanto, construir una teoría completa del derecho que tenga un aspecto justificador de las decisiones que adoptan las distintas instancias jurídicas. En este sentido la teoría será un auxilio indispensable para el que toma decisiones públicas. Para tomarlas se debe realizar una tarea de construcción y justificación. El científico del derecho, el filósofo del derecho y de la política no es un observador imparcial cuya función es describir el derecho y los valores, sino que es un constructor de soluciones, un especialista en la resolución de conflictos sociales. Desde esta perspectiva su intención es la construcción de modelos metodológicos que permiten solucionar problemas. Junto al aspecto descriptivo, Dworkin coloca el aspecto normativo, que es el que más interesa al profesional y al juez. La teoría orienta la práctica. Evidentemente, tanto el método como las soluciones han provocado una cascada de críticas y desacuerdos importantes. Pero me parece que uno de los grandes méritos de la polémica ha sido discutir problemas en vez de describirlos, y en este punto Dworkin ha sido un maestro.

Pero no voy a valorar todos los aspectos de su obra, sino simplemente algunos del método dworkiniano.

\section{Análisis del desacuerdo entre los juristas}

Una de las características fundamentales de la profesión jurídica es la controversia. Los juristas discuten y tienen muchos desacuerdos acerca de las soluciones que ofrece el derecho positivo. Ahora bien, muy pocas teorías han prestado atención al análisis de los 
desacuerdos. Dworkin sugiere que ciertamente existen muchos desacuerdos que no son misteriosos. Por ejemplo, pueden existir desacuerdos factuales, es decir sobre los hechos. Este desacuerdo es importante en el derecho pero el tipo de pruebas que necesitamos para que un hecho se considere probado es intersubjetivo. Si el presunto delincuente iba vestido de negro o no es una cuestión que puede ser relevante e incluso decisiva para probar una conducta delictiva. El tipo de evidencia que necesitamos puede ser muy variada, pero creo que los desacuerdos entre los juristas no se basan casi nunca en cómo probar los hechos, sino en una cuestión de hecho, es decir, en si con los datos disponibles esa conducta debe considerarse probada o no. Los juristas, con los datos disponibles, construyen historias que persuaden al juez de la coherencia de su argumento. Pero los desacuerdos factuales tienen pruebas intersubjetivas y, por tanto, sabemos qué necesitamos para convencer.

Otro de los desacuerdos hace referencia a las ideas de justicia. Si organizamos un debate sobre la eutanasia, el aborto o la justificación de la guerra seguramente existirán desacuerdos. Pero esos desacuerdos no son los que tienen normalmente los juristas. En realidad, nuestra sociedad es una sociedad pluralista y se basa en el supuesto según el cual los individuos tienen unas concepciones diferenciadas de la justicia. No hay forma de probar si una es mejor que la otra. No es ningún misterio que existan desacuerdos sobre lo que es incorrecto moralmente porque en realidad nuestra sociedad presupone la posibilidad del desacuerdo. Una sociedad democrática tiene unas reglas de juego que permiten ideologías distintas.

Otro tipo de desacuerdo puede ser sobre el significado de la norma. Muchos juristas creen que los desacuerdos provienen de la ambigüedad del lenguaje ordinario. La tarea del jurista es parecida a la de un filólogo. Como el lenguaje es ambiguo entonces se explica que existan controversias porque cada jurista construye su argumento fundamentándolo en una posibilidad interpretativa.

Dworkin sostiene que los principales desacuerdos que existen entre los juristas no son los desacuerdos puramente verbales y semánticos. No los niega, sino que señala que éstos no son los más relevantes. El derecho entendido como concepto interpretativo supone que los desacuerdos existentes entre los juristas no son sobre hechos ni sobre ideas de justicia, sino sobre lo que exige el derecho.

Pongamos un ejemplo. Supongamos que existe una ley que prohíbe circular a más de $120 \mathrm{Km}$. por las autopistas y que el ciudadano A circula a $160 \mathrm{Km}$. Un policía lo detecta con un radar 
fiable y le impone la multa. Con independencia de las ideas de justicia que tenga cada uno de los que intervienen parece ser que éste es un caso fácil. Los hechos están probados, la norma es vigente y la sanción no admite discusión. No hay desacuerdo crucial. Pero imaginemos el mismo caso, los mismos hechos y las mismas leyes. Supongamos que el ciudadano A va a un hospital y en la entrada se encuentra un médico amigo suyo que debe trasladarse urgentemente a otra ciudad para hacer un trasplante de riñón. Sólo si circula a $160 \mathrm{Km}$. salvará la vida del paciente. En la autopsia encontramos el policía que le pone la multa. Si en el primer caso estaba justificada la multa, en este segundo caso es más dudoso. Seguramente no encontraríamos la unanimidad del primer caso, y no hay que olvidar que los hechos son los mismos y las normas son las mismas. El desacuerdo no es sobre la semántica de las normas ni sobre los hechos, sino sobre lo que exige el derecho, sobre el fundamento que justifica la coacción del Estado. En el primer caso no se discute el fundamento de la norma, en el segundo sí. Ello conduce a discutir el papel que ejerce la justificación basada en razones, principios y directrices que pretenden garantizar el bienestar colectivo.

Existen desacuerdos sobre lo que el derecho exige. Este es el desacuerdo básico, el que se debe explicar, el que tiene quien trata el derecho desde el punto de vista interno. Los desacuerdos entre los juristas no provienen de que no están de acuerdo en si una ley ha sido dictada o no. Su desacuerdo es mucho más profundo porque hace referencia a la cuestión de hasta qué punto la coacción del estado debe utilizarse en este caso y cómo se debe justificar. El problema no es sólo la identificación del texto sino su uso. Y eso exige la reconstrucción de algo más complejo y abstracto que la semántica de la norma. Aunque estén de acuerdo sobre cuáles son las leyes vigentes, y cuál es el sentido de las palabras de la ley, puede existir, y existe, el desacuerdo teórico sobre lo que exige el derecho. Dworkin sugerirá que el derecho es un concepto interpretativo y que los textos por sí solos no nos dicen nada. Se necesita un enfoque determinado y eso es lo que el positivismo no ha apreciado. El concepto interpretativo del derecho es su arma más poderosa en el desafío al positivismo. 


\section{Dos preguntas}

\section{1. ¿Cómo determina el pasado al presente?}

Dworkin se plantea la cuestión de hasta qué punto el pasado determina el presente. Ésta es una pregunta que toda teoría resuelve. Y según cuál sea la respuesta podemos clasificar las doctrinas e incluso apreciar sus puntos más débiles.

Las doctrinas positivistas -por ejemplo- ofrecen una respuesta precisa a esta cuestión. Las decisiones del pasado -es decir, las leyes, los precedentes, las costumbres jurídicas-, constituyen los criterios mediante los cuales se deben fundamentar las decisiones del presente. Cuando existen leyes que tratan de los problemas que se plantean ante los jueces, éstos deben decidir conforme a los criterios preestablecidos. En el caso de que no exista una ley aplicable o un precedente, o una decisión del pasado, entonces el positivismo se divide. Kelsen afirmaría -por ejemplo- que el derecho siempre ofrece respuesta porque el juez debe rechazar la demanda cuando no encuentra en el derecho una norma aplicable. La norma general excluyente tiene la función de cerrar el sistema. Ésta sería la posición más fuerte del positivismo, ya que mantiene que las decisiones del pasado determinan el presente (Kelsen, 1960, epígrafe 34, g). Los jueces y demás órganos decisorios están sujetos a las normas jurídicas y a las decisiones explícitas del pasado. Cuando no hay decisión explícita entonces no existe discreción judicial. El juez también aplica el derecho cuando rechaza la demanda.

Una posición más moderada será la defendida por Hart. La completitud del derecho no es una propiedad necesaria del mismo. El juez se puede encontrar con lagunas y en este caso puede decidir conforme a su discreción (Hart, 1961, págs. 138 y ss.). En principio los jueces están determinados por las decisiones del pasado siempre y cuando estas decisiones se hayan producido. Si no hay decisión previa entonces el juez no se ve limitado y decide discrecionalmente.

Dworkin llama convencionalismo a esta posición. El derecho se encuentra en las convenciones del pasado. El pasado determina el presente. El valor subyacente está claro. La fidelidad a la ley supone la garantía de la seguridad jurídica. El convencionalismo estricto responde que el pasado siempre determina el presente, mientras que el convencionalismo moderado responde afirmativamente a la cuestión aunque reconoce la discrecionalidad judicial para el caso en el que no exista convención. Sobre este punto volveremos después. Podemos dejar de momento el problema aquí. 
Frente al convencionalismo nos encontramos con el pragmatismo. Una variante del pragmatismo es la doctrina realista. A la cuestión «cómo determina el pasado el presente» el realismo contesta con afirmaciones contundentes. Los jueces no deben quedar limitados por las decisiones del pasado sino que tienen que administrar justicia. Los pragmáticos contestan que el pasado no determina el presente. Los jueces deben guiarse por el criterio de justicia y muy especialmente por el criterio del bienestar general. Las leyes no son nada más que juguetes vistosos que los jueces utilizan a su antojo. Lo primordial es la solución justa; lo secundario es cómo se justifica conforme a las decisiones del pasado. Las posiciones realistas y pragmáticas parten del presupuesto de que el juez tiene conciencia de la rectitud y trata de encontrar lo que es justo para la solución del caso. Una vez encontrada la solución, acude a las normas como criterio de justificación. La intuición y el resultado justo son los criterios decisivos que orientan su actividad ${ }^{2}$.

Tenemos dos modelos de doctrina que responden a la cuestión planteada: Los jueces ¿crean o aplican normas? Los convencionalistas contestan que el juez no crea derecho, que el asunto del derecho es de convenciones producidas en el pasado. Los pragmáticos contestan a la cuestión sugiriendo que la respuesta es negativa. El pasado no determina el presente. Las convenciones del pasado tienen menos relevancia que la conciencia de la rectitud, que la idea de la justicia del caso (Larenz, 1980, pág. 198 y ss. y G. Carrió 1979).

\section{2. ¿Cómo se justifica la coacción del estado?}

Esta cuestión depende de cuál sea la respuesta a la primera pregunta. El convencionalista sostendrá que la coacción se justifica porque las convenciones del pasado la prevén. Una sociedad democrática basa la justificación de la coacción en la voluntad popular. El juez aplica el derecho y la coacción está justificada porque las decisiones están fundamentadas en normas. Sin embargo, el convencionalista moderado -que es el más sutil y el más sofisticado- tendrá dificultades a la hora de justificar la discreción judicial, porque supone la utilización de la coacción del

${ }^{2}$ Esta posición favorece filosofías políticas de carácter utilitarista aunque no todo utilitarista es pragmático. Sobre este problema véase H. L. Polhman: Justice, Oliver W. Holmes and Utilitarian Jurisprudence, Harvard University Press, 1984. 
estado sin estar fundamentada en decisiones anteriores. Por tanto, la soberanía popular no las legitima ${ }^{3}$.

El pragmático ofrece una respuesta diferente. La decisión judicial es el fundamento de la coacción del estado. Las decisiones del pasado no son relevantes. Lo único relevante es si las decisiones mejoran el bienestar social. Los jueces son los órganos del estado más adecuados para tomar las decisiones que producen directamente unos resultados más justos. El modelo pragmático ofrece un compromiso con la justicia como sustitutivo de cualquier procedimiento que limita la persecución de los resultados más justos. Como consecuencia de ello la coacción del estado está justificada siempre y cuando se alcancen resultados justos ${ }^{4}$.

\section{¿Por qué estas respuestas son insatisfactorias?}

Dworkin se enfrenta a las dos respuestas ofrecidas por el convencionalismo y el pragmatismo. El problema principal de las dos concepciones es que no se toman los derechos en serio. Además dotan al juez de un poder creativo de derecho sin legitimación suficiente. El convencionalismo -al admitir la discreción judicial- y el pragmatismo al perseguir el resultado más justo violan algunos principios fundamentales de la sociedad democrática como son la irretroactividad de las leyes, la seguridad jurídica, la certeza del derecho y la inalienabilidad de los derechos. Ningún bienestar colectivo, ni ningún resultado colectivo beneficioso puede fundamentar la violación de un derecho. Los derechos triunfan frente al bienestar colectivo. Tanto el convencionalismo como el pragmatismo ofrecen respuestas contradictorias pero con defectos semejantes. El derecho ni se encuentra en las decisiones del pasado ni tampoco en las intuiciones de lo que es la justicia. El derecho en una sociedad democrática es algo más complejo que lo que estas dos visiones nos ofrecen y es preciso realizar una empresa constructiva para entender por que ni el convencionalismo

\footnotetext{
${ }^{3}$ Desde el punto de vista de la legitimación la posición de Kelsen es más coherente que la de Hart.

${ }^{4}$ El realismo americano confió en la percepción de la justicia del juez. El substrato utilitarista favorecería la búsqueda del criterio que favoreciera la mayor felicidad para el mayor número posible o principios como el del mínimo coste social. Sobre este punto véase la crítica de B. Ackerman: Del Realismo al Constructivismo jurídico. Barcelona, Ariel, 1988, págs. 13 y ss.
} 
ni el pragmatismo ofrecen respuestas aceptables. 


\section{Un modelo de Sociedad Democrática}

Dworkin ha desbrozado el camino para inaugurar su tarea constructiva. Propone operar con un modelo ideal de una sociedad democrática. La construcción del modelo no pretende reproducir la realidad sino formular cuestiones desde el modelo para llegar a comprenderla mejor. El puente entre la Ciencia de la Legislación y la Jurisprudencia se empieza a construir.

Una sociedad democrática -sugiere Dworkin- es una sociedad en la cual se respetan los siguientes principios.

Primero.- La Equidad: Entenderemos por equidad el valor del igual poder de cada uno de los individuos en esta sociedad. Una de las especificaciones más relevantes del principio de equidad es que cada uno debe contar como uno y nada más que uno (Dworkin, 1986, págs. 165 y ss.). El principio de dar a cada persona un voto es un principio equitativo.

Segundo.- Justicia: La Justicia -según Dworkin- se refiere a los resultados que producen las decisiones. Calificaremos una decisión como justa si asigna y distribuye los recursos conforme a un modelo ideal determinado. La equidad se refiere al procedimiento y a la imparcialidad; la justicia a los resultados. La distinción conceptual es importante porque se pueden dar casos de decisiones justas con procedimientos no equitativos y decisiones injustas con procedimientos equitativos. Sobre este punto volveremos más adelante.

Tercero.- Principio de Legalidad: En una sociedad democrática sólo se admite como legítimo el poder juridificado. El poder estatal debe funcionar por los cauces jurídicos. Una sociedad bien diseñada no admite policías paraestatales ni el uso de la fuerza no jurídica. El derecho distribuye la fuerza colectiva, es él mismo la organización de la fuerza. Un buen derecho democrático debe canalizar el ejercicio de la fuerza a través de cauces preestablecidos.

Cuarto.- Integridad: Una sociedad democrática está bien diseñada si responde a la virtud de la integridad. Una primera intuición de lo que Dworkin entiende por integridad puede expresarse acudiendo el lenguaje ordinario. En el lenguaje natural decimos que una persona es íntegra moralmente cuando obra conforme a principios. La virtud de la integridad supone no sólo la existencia de unos principios, sino también su organización coherente. Dworkin sugiere tratar a la comunidad como si fuera una persona moral ${ }^{5}$.

\footnotetext{
${ }^{5}$ Véase la entrevista de Juan R. Páramos a Hart en Doxa 1988, en la cual
} 
Con esto basta para tener una primera idea sobre el modelo que legitima la coacción del estado. Si una comunidad política se acerca a los valores de equidad, justicia, debido proceso e integridad, resultará que la coacción estará justificada, mientras que si se aleja de ellos entonces no estará justificada.

Pero vayamos por pasos. ¿Por qué es necesaria tanta discusión conceptual? Estos conceptos son distintos y conviene no confundirlos.

Vamos a ver la diferencia entre equidad y justicia. Prima facie podríamos afirmar que un procedimiento equitativo tiende a producir resultados justos. Una primera intuición nos muestra que si garantizamos la equidad de los procedimientos se alcanzan resultados justos. Sin embargo, eso no es cierto, porque es posible que un procedimiento no equitativo produzca resultados justos y un procedimiento equitativo produzca resultados injustos.

Pongamos ejemplos para aclarar la distinción. Supongamos que cuatro personas -tres chicos y una chica- deciden constituir una sociedad para hacer deporte. Cada una de ellas es propietaria de un 25 por 100 del capital y por unanimidad diseñan unos estatutos en los cuales cada uno tiene un voto y las decisiones se toman por mayoría. La primera decisión importante es comprar un campo y construir una pista de tenis. Se adopta la decisión por unanimidad. Una vez realizada la operación, los socios se reúnen para redactar un reglamento de uso de la pista. Los tres chicos deciden por mayoría que la chica no puede jugar nunca. El procedimiento es equitativo, y, sin embargo, el resultado que produce es manifiestamente injusto. Este es un contraejemplo a la tesis «meramente procedimental» de la justicia. Se necesita alguna cosa más que el procedimiento equitativo para que la decisión sea correcta.

Pero -al mismo tiempo- sería posible un procedimiento no equitativo que produjera resultados justos, como por ejemplo que decidiera uno solo y que el contenido de la decisión fuera que todos tienen las mismas oportunidades de jugar y que, por tanto, la chica tiene los mismos derechos que los otros. En este caso tenemos un procedimiento no equitativo que produce un resultado justo.

Hart afirma que el principal logro de Dworkin «consiste en haber demostrado cómo gran parte de los sistemas jurídicos ingleses y americanos se pueden analizar fructíferamente como si cada uno de estos sistemas fuera el resultado de un único autor coherente y consistente, preocupado por llevar a cabo en el Derecho una específica moralidad política singularmente liberal, en el sentido de que trata a todos los individuos como moralmente legitimados para una igual consideración, considerado a los derechos básicos individuales como elementos prioritarios sobre los valores colectivos. 
Este modelo ideal nos empieza a inquietar porque permite vislumbrar algunos de los problemas de la legitimación de la democracia, ya que no sólo debemos exigir que el procedimiento sea equitativo, sino que también produzca un resultado suficientemente justo para que pueda justificar la coacción del estado. La distinción conceptual es necesaria y nos alerta frente a legitimaciones procedimentales. Nos avisa que una comunidad no sólo se justifica por el procedimiento, sino también por el contenido de su derecho (Barry, 1989, págs. 24 y ss.).

\section{Integridad, equidad y justicia}

El principio de legalidad lo dejaremos aparte, ya que no ofrece especiales problemas para nuestro argumento. Nuestro tema ahora es ver las diferencias entre equidad, justicia e integridad.

Empecemos con un ejemplo. Supongamos que varios amigos van a cenar a un restaurante y que algunos desean pescado y otros carne. Parece ser que una de las soluciones mejores -tanto desde el punto de vista de la equidad como de la justicia- (si es que se puede hablar de ello en este caso) es que cada uno decida según sus preferencias. No hay ninguna razón -a igualdad de precios- que justifique elegir el mismo plato en el restaurante. Una decisión de este tipo la podríamos denominar diversificada. Esta decisión parece respetar el principio de equidad y el de justicia.

Sin embargo, ¿por qué cuando tratamos de la actividad legislativa del estado no admitimos que las leyes estén diversificadas? Supongamos que el parlamento respeta fielmente la voluntad popular y que se propone una ley sobre la eutanasia activa. El 45 por 100 de los diputados vota en contra y el 55 por 100 a favor. No se produce ninguna abstención ni ningún voto nulo. ¿Sería mejor aceptar una ley que permitiera la eutanasia activa en el 55 por 100 de los casos y la prohibiera en el 45 por 100, o sería preferible una ley unificada que siempre la prohibiera o la permitiera?

Veamos qué es lo que dice nuestro modelo. Vayamos a la equidad. Es indudable que una ley diversificada es más equitativa que una unificada. La razón es muy sencilla, porque cada uno está igualmente representado en la ley diversificada mientras que la minoría perdedora no está en ningún caso representada en la ley unificada. De hecho defender el punto de vista de la ley unificada supone convertir el 55 por 100 de los votos en el 100 por 100. La voluntad de los 55 vale por los 100. Una ley diversificada cumple mejor con el requisito de equidad. Por tanto no hay razones de equidad para oponernos a esta decisión. 
Una vez despejada esta incógnita vayamos a la segunda. ¿Una decisión diversificada es contraria a la justicia? Para responder a esta cuestión es necesario ponerse de acuerdo acerca de lo que entendemos por justicia. Una de las características fundamentales de una sociedad democrática es el pluralismo ideológico, lo cual significa que existen diversas concepciones de la justicia contradictorias. Los resultados que a unos les parecen justos a otros les parecen injustos. No es ningún misterio que existan desacuerdos sobre la justicia. Seguramente una ley diversificada responde mejor a las concepciones individuales de justicia que una unificada. Si nos guiamos por el resultado del que pierde la votación veremos que es mejor desde su perspectiva que en vez de producirse 100 injusticias se produzcan 45. Desde el punto de vista del que pierde no hay razones de justicia para defender las leyes unificadas. Desde el punto de vista del que gana es evidente que la ley unificada responde a su concepción de la justicia. Pero ello supone el triunfo de una concepción de la justicia sobre la otra y atenta contra el pluralismo.

Tanto desde el punto de vista de la equidad como -en menor medida- desde el punto de vista de la justicia no hay razones muy poderosas para oponerse a las leyes diversificadas. Sin embargo, tenemos la intuición de que una ley diversificada atenta contra principios importantes. El problema de la eutanasia es cualitativamente distinto al de la cena. Lo que nos lleva a negar las leyes diversificadas es la virtud de la integridad. Concebimos que una sociedad democrática no sólo debe respetar la equidad y la justicia -tal y como las hemos definido-, sino que esa comunidad no es una comunidad cualquiera, sino una comunidad regida por un conjunto de principios consistentes. Una sociedad democrática supone una cierta personificación de la comunidad y una concepción colectiva coherente de la justicia. Es lo que podríamos denominar una concepción de la justicia institucional (Dworkin, 1986, págs. 225 y ss.).

La virtud de la integridad exige, por tanto, que las leyes no sean el fruto de un compromiso entre concepciones de justicia subjetivas contradictorias, sino que respondan a una concepción coherente de la justicia pública. Una sociedad democrática requiere -por tanto- que los actos de coacción estatal estén justificados conforme a principios. Supone tratar la comunidad como si fuera una persona moral y exigir a esa persona integridad moral.

Pero inmediatamente se plantean problemas importantes. ¿Qué quiere decir que una comunidad se personifique? ¿Qué quiere decir una comunidad de principios? Dworkin vuelve a los modelos. Sabe perfectamente que la sociedad no es una persona, pero 
sugiere que la concepción del derecho como integridad más que una realidad es un enfoque. Si tratáramos a las comunidades políticas estatales como unas comunidades de principios, tendríamos elementos suficientes para observar hasta qué punto las decisiones del pasado determinan el presente y hasta qué punto las decisiones que toman los poderes públicos que afectan a la coacción están justificadas. El esfuerzo constructivo supone partir de una intuición determinada -como por ejemplo la de que no están justificadas las leyes diversificadas- y preguntarse cuáles son los principios, o la estructura coherente de principios, que mejor justificarían las decisiones públicas. El paso siguiente sería considerar estas decisiones como los eslabones de una cadena -la cadena del derecho. Para separarse de las decisiones anteriores es preciso que existan razones públicas para decidir en un sentido determinado.

Pero esta comunidad de principios ¿constituye una especie de caja de caudales trascendental? ¿Es otra forma de iusnaturalismo? Dworkin en este punto es taxativo. Las valoraciones y los principios que defiende colectivamente una sociedad son dinámicos. Quizá los nombres sean los mismos, pero su significado varía a lo largo de la historia. El derecho como integridad es un enfoque que permite al ciudadano una actitud activa frente al derecho. Le recomienda que tome el derecho como un dato interpretativo y que colabore en la construcción de la tarea colectiva de justificación y crítica de las decisiones públicas. El derecho para Dworkin no es un producto acabado ni tampoco es la justicia. Es algo menos y algo más. Es algo menos que la justicia porque la coherencia -es decir, la virtud de la integridad- exige sacrificar en algunas ocasiones la persecución de los resultados justos. Es algo más que las convenciones porque el derecho está compuesto no sólo por un conjunto de normas, sino también por un conjunto de principios ${ }^{5 \text { bis }}$.

\section{Los principios y el derecho}

Poco a poco, Dworkin nos ha ido introduciendo en un mundo que difiere sustancialmente del derecho visto desde la perspectiva positivista. El diseño de una sociedad democrática ideal supone la

5bis Dworkin distingue entre integridad en la legislación y en la adjudicación. También distingue entre integridad institucional e integridad pura. En los últimos trabajos sobre el sentido de la vida -todavía no publicados- la noción de integridad la ha utilizado para la conceptualización de las personas. 
aceptación de la virtud de la integridad. La virtud de la integridad supone que la comunidad como tal es una comunidad que tiene un sustrato de principios que la justifica. Una decisión pública está justificada no sólo si respeta la equidad y la justicia, sino si además respeta la coherencia de principio. La integridad moral de la comunidad es análoga a la integridad moral de la persona.

El paso siguiente será considerar que el derecho no sólo está compuesto por normas sino también por directrices y principios. Existen unas diferencias lógicas entre las normas y los principios que conviene resaltar (Dworkin, 1977, págs. 22 y ss.). Según Dworkin las normas o se aplican o no se aplican. No cabe una tercera vía, mientras que en el caso de los principios la cuestión no es clara.

Las normas establecen ellas mismas sus condiciones de aplicación, mientras que los principios dan razones para decidir en un sentido determinado pero no establecen sus propias condiciones de aplicación.

Otra diferencia importante entre las normas y los principios es que aquéllas se pueden identificar mediante un test de origen mientras que éstos no. Sabemos si una norma legislativa pertenece a un ordenamiento determinado si se puede deducir su validez de la constitución, mientras que no se puede deducir la validez de un principio por su origen, sino por su fuerza argumentativa, es decir, por su peso específico ${ }^{6}$.

Una tercera diferencia importante se encuentra en el criterio de distinción entre una norma jurídica y una no jurídica: el tipo de sanción específico. No existen policías ni cárceles morales, mientras que en las sanciones jurídicas la coacción está organizada e institucionalizada. Pues bien, los principios no se pueden distinguir por la forma de la sanción. Lo que les caracteriza es un contenido determinado, una fuerza argumentativa. Y aquí la distinción entre principios jurídicos y los principios sociales o morales no es posible, ya que la moral no se distingue del derecho por su contenido, pese a los intentos de filósofos de encontrar en este criterio material una vía de solución al problema de la separación entre derecho y la moral (Greenawalt, 1987).

${ }^{6}$ Véase la crítica de G. Carrió a Dworkin en las obras citadas en las que sostiene la posibilidad de ampliación de la regla de reconocimiento para la identificación de los principios. 


\section{Principios morales y jurídicos}

A medida que nos introducimos en el mundo de los principios vamos descubriendo que es imposible distinguir un principio jurídico de un principio moral o social. La única diferencia reside en la posible utilización por parte de los tribunales. Pero nada impide que un principio moral no reconocido jurídicamente sea reconocido en el futuro. El caso de abuso de derecho es un buen ejemplo que demuestra esta tesis. No se debe olvidar que el problema que interesa resolver al usuario del derecho no es cómo se resolvieron los problemas, sino cómo se resolverán. Y no está excluido que se aporte nuevas soluciones para resolver viejos problemas.

\section{Concepto y concepción}

Dworkin sostiene que el derecho no sólo está compuesto por normas, sino también por directrices -que tienen como objetivo perseguir un bien colectivo- y principios -que son razones para decidir en un sentido determinado.

Los principios acostumbran a ser de carácter muy general. Un mismo principio puede dar origen a desacuerdos entre los juristas a la hora de interpretarlo. Dworkin recomienda que en el tratamiento de los problemas jurídicos adoptemos el enfoque de la integridad, es decir, que deberíamos tratar el ordenamiento jurídico como si fuera el producto de una persona coherente e íntegra moralmente. En nuestra constitución podemos encontrar ciertos principios de carácter muy general -como, por ejemplo, el principio de igualdad- que no sirven sin un criterio material de determinación. El principio de igualdad formal supone el tratamiento igual de lo que es igual y desigual de lo que es desigual. Éste constituye el concepto de igualdad. Sin embargo, este concepto común a todas las concepciones de la igualdad no sirve para dirigir la conducta humana, pues existen diversas concepciones que determinan lo que es igual y lo que es desigual. Por ejemplo, en nuestras sociedades se utiliza el criterio del mérito, el del resultado y el de las necesidades, entre otros. Cada uno de esos criterios especifica el concepto formal de igualdad y produce resultados distintos en su aplicación.

El enfoque del derecho como integridad no sería escéptico respecto a estas aparentes contradicciones y trataría de indagar cuáles son las razones que convierten un criterio como el del mérito en adecuado para solucionar determinados problemas, 
mientras que, en cambio, para otros esta sociedad utiliza el criterio de las necesidades. El derecho como integridad trata de reconstruir la historia. Allí donde se encuentra con criterios contradictorios trata de encontrar una explicación y trata, por tanto, de exigir que las distinciones entre criterios no se produzcan al azar, sino que respondan a razones públicas y justificadas.

Pero al mismo tiempo esta construcción teórica permite formular propuestas de cómo se deberían resolver los problemas en el caso de que existan problemas de coherencia. El derecho como integridad es un enfoque que trata los problemas jurídicos desde la mejor perspectiva posible, no se conforma con suponer la irracionalidad, sino que exige que la actividad coactiva del estado responda a un conjunto de principios coherentes. Y lo busca.

Sus armas conceptuales son sofisticadas, pero parte de intuiciones muy sencillas. Ahora bien, su método parte de la intuición, pero sólo se acepta si es coherente con un conjunto de principios. En caso contrario se rechaza. El intuicionismo sólo es el punto de partida. La coherencia con la textura de principios de la comunidad es la prueba que debe pasar la intuición para aceptarse e integrarse en el sistema. Además del test de adecuación al pasado, la integridad exige el paso de un segundo test de justificación normativa institucional ${ }^{6 \text { bis }}$.

Las dos preguntas fundamentales de Dworkin, ¿cómo determina el pasado el presente? y ¿cómo se justifica la coacción?, se responden en base a la concepción del derecho como integridad. Un estado es una comunidad de principio y el conjunto de principios del pasado determina el presente, pero no absolutamente sino sólo relativamente. El derecho como integridad es un enfoque que exige justificar mediante razones el abandono de principios anteriores. La integridad no tolera la irracionalidad. Las normas que componen un ordenamiento jurídico están sostenidas en un conjunto de principios justificadores. El elemento fundamental del derecho no es la norma sino el principio justificador. La fidelidad a la ley es una fidelidad al conjunto de principios que identifican la comunidad. Ahí reside su gran diferencia con el positivismo. Obsérvese que las cuestiones jurídicas son cuestiones de justificación de decisiones. Ese es su punto de vista.

Pero, frente al realismo -que persigue la justicia sin equidad, sin procedimiento y sin coherencia-, el enfoque del derecho como integridad exige limitaciones a la persecución del resultado de justicia. La justicia no es el único valor importante en el

${ }^{6 b i s}$ Ambos test están profundamente relacionados y existen interacciones entre ellos. 
derecho. Junto a él existen otros como la equidad, el proceso debido, el principio de legalidad y la coherencia. El pasado -y en ese pasado los principios juegan un papel crucialdetermina el presente en el derecho como integridad. El realismo es un experimento que privilegia valores directamente sin ningún tipo de limitación institucional. Además, el realismo tiende a garantizar el bienestar general bajo el influjo de la filosofía política utilitarista.

La segunda cuestión, ¿hasta qué punto está justificada la coacción del estado?, la responde Dworkin mostrando un modelo de equilibrio entre los valores de la democracia. Por supuesto que no existen democracias reales que coincidan plenamente con el modelo. Sin embargo, en la medida en que se acerquen se justificará la coacción del estado.

\section{Reflexiones críticas}

Las dos mejores aportaciones de Dworkin a la filosofía jurídica son: la concepción del derecho como interpretación y el enfoque que proporciona el valor de la integridad. Sin duda, el derecho como concepto interpretativo es el arma más poderosa contra el positivismo y constituye el primer peldaño de su construcción. El segundo es el enfoque del derecho desde la perspectiva del concepto de integridad. Este enfoque ha producido numerosas tesis originales que se oponen a la visión utilitarista. El tercero, y en eso ha seguido a Hart, vincula el estudio del derecho al pensamiento filosófico. En el caso de Dworkin, la filosofía del segundo Wittgenstein, Rawls y últimamente la Hermenéutica y la crítica literaria.

No quisiera acabar sin apuntar algunas críticas a las ideas dworkianianas. En primer lugar, el enfoque de la integridad puede tener un valor absoluto o relativo. En ciertos pasajes, Dworkin sugiere que el valor de integridad es uno más entre otros, mientras que a veces parece que es ciertamente el valor supremo. La racionalidad y la coherencia del sistema de principios sería más importante que la justicia o la equidad. Eso significa que la integridad tiene fuerza para restringir las exigencias de la equidad o de la justicia. En su teoría no queda suficientemente claro el papel que juega la integridad y cuándo triunfa. La idea de integridad es muy ambigua, porque no sabemos si la justicia está subordinada a ella o no. Parece ser que el intérprete tiene discreción para justificar su decisión en base a este principio o cualquier otro importante.

Tampoco queda muy claro por qué el pasado debe ejercer un papel tan preponderante. Dworkin dota de un valor excesivo al 
principio de estabilidad. Pienso que los principios que deben aplicar los tribunales deben ser los más adecuados para resolver los conflictos, y no los que se adecúen mejor a la historia institucional. No se entiende muy bien por qué los jueces deben seguir los principios institucionales históricos y no los más correctos (Barek, 198 1, pág. 31). Además, en una tradición rica como la de los países desarrollados siempre se pueden encontrar fundamentos históricos para las decisiones contradictorias (Posner, 1990, págs. 202 y ss.). En la práctica es difícil establecer prioridades absolutas. Sin ellas no hay respuestas correctas.

Es cierto que si Hércules tiene tiempo ilimitado, inteligencia portentosa e información completa, será capaz de ofrecer una buena teoría para resolver problemas ${ }^{7}$. Pero otro juez ideal ¿acaso no podría ofrecer una respuesta distinta? ¿Cuál es el criterio que permite resolver la disputa? Los dos pretenden que su respuesta es la correcta. Pero ese ideal regulativo que debe presidir toda actividad judicial no se puede tomar al pie de la letra. El mundo real es distinto al mudo ideal de la información completa y tiempo ilimitado. Las tesis de Hércules sólo serían correctas si en el mundo real se cumplieran estas condiciones, y al no cumplirse no es posible enunciar la teoría de la respuesta correcta. Pero no sólo eso, pues es posible que cumpliéndose esas condiciones no se llegue a una solución definitiva y que el desacuerdo se mantenga ${ }^{8}$. Lo que sí es cierto es que quien toma una decisión pública argumenta que su plan es el mejor e intenta justificarlo. Pero ese es el ideal hacia el que tiende quien toma una decisión pública, y no es posible confundir la explicación de la intención del que decide con su resultado. Sólo con mucha cautela se puede pasar del mundo ideal al mundo real, y no existen garantías de una objetividad como pretende

${ }^{7}$ Véase R. Alexy, «Sistema jurídico, principios jurídicos y razón práctica», en Doxa 5, 1988, pág. 151, en la que afirma: «Una única respuesta correcta sólo podría admitirse recurriendo a cinco idealizaciones: 1) tiempo ilimitado, 2) información ilimitada, 3) claridad lingüística ilimitada, 4) capacidad y disposición ilimitada para el cambio de roles, 5) carencia de principios ilimitada». Véase también los recientes trabajos de L. Barragán, «La respuesta correcta única y la justificación de la decisión jurídica» y de A. Aarnio, «La tesis de la única respuesta correcta y el principio regulativo del razonamiento jurídico» en Doxa 1990.

${ }^{8}$ Como afirma Alexy, ibid. pág. 151, «queda aún por ver si sobre la base de semejantes idealizaciones es posible una única respuesta correcta para cada caso o si las diferencias antropológicas de los participantes en el discurso que obstaculizan el discurso hacen que, pese a las idealizaciones, puedan existir casos sin una única respuesta correcta. En todo caso, está claro que en la realidad no existe ningún procedimiento que permita, con una seguridad intersubjetivamente necesaria, llegar en cada caso a una única respuesta correcta». 
Dworkin. 
Otra cuestión muy enlazada con la anterior. La personificación de la comunidad y la justicia institucional. Aquí Dworkin está introduciendo una serie de criterios comunitaristas que no se compaginan muy bien con su idea de que los derechos son individuales. La aceptación de esa ficción comunitarista no es necesaria y constituye una especie de hipótesis ad hoc para defender un principio de solidaridad insuficientemente fundamentado. La defensa de una justicia institucional constituye el fundamento de por qué las respuestas son correctas (Posner, 1990, págs. 202 y ss.). Pero no hay acuerdo sobre el contenido de la justicia institucional (Atienza, 1991).

Otro aspecto interesante es preguntarse por qué los jueces deben decidir conforme a principios y no conforme a directrices que tiendan a preservar el bien colectivo. En los ordenamientos jurídicos positivos no existe ninguna regla que obligue a los jueces a juzgar conforme a los principios. Un Hércules II podría defender la idea de que los casos difíciles no sólo se deben resolver conforme a los principios que históricamente definen a esa comunidad, sino conforme a objetivos, directrices políticas, estándares y principios vigentes en esta sociedad. Si eso se acepta, y entonces el juez ideal tiene discreción, ¿cuál es la mejor respuesta? Esa no es una cuestión que se pueda contestar sin una toma de posición filosófica.

Para acabar, en líneas generales se puede afirmar que las intenciones del positivismo y de Dworkin son muy distintas. El positivismo jurídico pretende describir el derecho como un producto acabado compuesto por una variedad de elementos (Raz «Legal Principles and the Limits of Law», en Cohen, 1984, págs 87 y ss.). No está -en principio- interesado en el uso y en la justificación de las decisiones, sino en la identificación de los elementos y los materiales de construcción del argumento jurídico. Por esto, y desde su paradigma, el positivista estará muy interesado en distinguir entre el derecho y la moral. Se preocupa por diseñar criterios de delimitación de lo jurídico. Estudia el derecho como un producto acabado preparado para usarse en la resolución de conflictos. Pero el positivismo tiene que dar el salto -y en eso no es coherente- del mundo de la descripción al mundo de la justificación 9 .

El planteamiento de Dworkin es muy distinto. Su preocupación fundamental no es la descripción sino la justificación. Pretende construir una teoría que sirva para ser usada por jueces y tribunales. Dworkin no estudia el derecho desde fuera, intentándolo describir

\footnotetext{
${ }^{9}$ Véase la entrevista de Raz en Doxa 9. La obra de Raz trata de responder a este problema.
} 
como algo estático, sino que ve el derecho desde la perspectiva interna del juez ${ }^{10}$.

Pienso que la polémica con el positivismo está desenfocada porque sus objetivos son muy distintos. Pero ese desenfoque ha sido especialmente útil para poner de manifiesto que el derecho debía ser observado desde la perspectiva del usuario y del que toma decisiones con fuerza vinculante. $\mathrm{Y}$, desde el punto de vista positivista, es muy difícil mantener las tesis de la independencia entre la moral y el derecho, la descripción y la prescripción y, al mismo tiempo, ofrecer una teoría útil para el operador jurídico.

Dworkin ha sido injusto con el positivismo porque sólo subrepticiamente el positivismo ha mantenido tesis fuertes sobre la adjudicación. En realidad, los positivistas han respondido a Dworkin con concesiones que no alteraban el contenido esencial del positivismo, como por ejemplo la ampliación de la regla de reconocimiento o bien admitiendo que la discreción judicial tenía sus límites.

El aspecto positivo más importante de la polémica ha sido colocar en el centro de la discusión el papel de los principios y valores a la hora de justificar decisiones. No es casual que -desde perspectivas muy distintas- la actual problemática metodológica se centre más en las cuestiones de justificación que en las cuestiones de descripción de las normas y de los valores jurídicos.

\section{BIBLIOGRAFÍA}

Atienza: «La huelga de hambre de los GRAPO». Claves de Razón Práctico, 1991, n. ${ }^{\circ} 14$.

Barek: Judicial Discretion. Yale University Press, 1981.

Barry: Democracy Power and Justice. Oxford Clarendon Press, 1989.

Cohen: Ronald Dworkin and Contemporary Jurisprudence. London,

Duckworth, 1984.

Dworkin (ed.): La Filosofía del Derecho. México, F.C.E., 1980.

Dworkin: A Matter of Principle. Harvard University Press, 1985.

Dworkin: Law's Empire. Belknap Press, 1986.

Dworkin: Taking Rights Seriously. Harvard University Press, 6 ed., 1977.

${ }^{10}$ Como afirma Raz, el derecho se concibe como la moralidad institucional de los tribunales. Véase Legal Principles and the Limits of Law, pág. 84. 
Greenawalt: Conflicts of Law and Morality. Oxford Clarendon Press, 1987.

Hart: The Concept of Law. Oxford Clarendon Press, 1961.

Kelsen: Reine Rechtslehre. Wien, F. Deuticke, 1960.

Larenz: Metodología de la Ciencia del Derecho. Barcelona, Ariel, 1980.

MacCormick: Legal Right and Social Democracy. Oxford Clarendon Press, 2. ed., 1984.

Oppenheim: Political Concepts. A Reconstruction. Harvard University Press, 1981.

Posner: The Problems of Jurisprudence. Harvard University Press, 1990. 